\title{
Low Intensity Focused Ultrasound Augmented Multifunctional Nanoparticles for Integrating Ultrasound Imaging and Synergistic Therapy of Metastatic Breast Cancer
}

\author{
Qian Zhang \\ Ningxia Medical University \\ Wen Wang \\ General Hospital of Ningxia Medical University \\ Hongyuan Shen \\ Ningxia Medical University \\ Hongyu Tao \\ Ningxia Medical University \\ Yating Wu \\ General Hospital of Ningxia Medical University \\ Liyuan Ma
}

General Hospital of Ningxia Medical University

Guangfei Yang

General Hospital of Ningxia Medical University

Ruijiao Chang

General Hospital of Ningxia Medical University

Jiaxing Wang

Ningxia Medical University

Hanfei Zhang

Ningxia Medical University

Chenyu Wang

Ningxia Medical University

Furong Zhang

Ningxia Medical University

Jiaojiao Qi

Ningxia Medical University

Chengrong Mi ( mcr69333@163.com )

Ningxia Medical University https://orcid.org/0000-0002-9843-2930 


\section{Nano Express}

Keywords: nanoparticle, sonodynamic therapy, contrast enhanced ultrasound, anti-metastasis, LIFU

Posted Date: January 22nd, 2021

DOI: https://doi.org/10.21203/rs.3.rs-151083/v1

License: (c) (i) This work is licensed under a Creative Commons Attribution 4.0 International License. Read Full License 


\section{Abstract}

The metastasis of breast cancer is believed to have a negative effect on its prognosis. Benefiting from the remarkable deep-penetrating and non-invasive characteristics, sonodynamic therapy (SDT) demonstrates a whole series of potential leading to cancer treatment. To relieve the limitation of monotherapy, a multifunctional nanoplatform has been explored to realize the synergistic treatment efficiency. Herein, we establish a novel multifunctional nano-system which encapsulates chlorin e6 (Ce6, for SDT), perfluoropentane (PFP, for ultrasound imaging), and docetaxel (DTX, for chemotherapy) in a well-designed PLGA core-shell structure. The synergistic nanoparticle (CPDP NPs) featured with excellent biocompatibility and stability primarily enables its further application. Upon low intensity focused ultrasound (LIFU) irradiation, the enhanced ultrasound imaging could be revealed both in vitro and in vivo. More importantly, combined with LIFU, the nanoparticle exhibits intriguing antitumor capability through Ce6 induced cytotoxic reactive oxygen species as well as DTX releasing to generate a concerted therapeutic efficiency. Furthermore, this treating strategy actives a strong anti-metastasis capability by which lung metastatic nodules have been significantly reduced. The results indicate that the SDT-oriented nanoplatform combined with chemotherapy could be provided as a promising approach in elevating effective synergistic therapy and suppressing lung metastasis of breast cancer.

\section{Introduction}

Breast cancer has haunted women for years as one of the most threatening malignant tumors. Due to the high heterogeneity and high metastatic ability, it is reported that the distant metastasis of breast cancer accounted for more than $90 \%$ of its mortality, whereas the 5 -year survival rate of advanced or metastasized patients is only $26 \%$, resulting in a poor clinical outcome [1-3]. The negative characterization of breast cancer has made it hard to be completely cured, promoting the treating strategy to be more challenging in eliminating the primary tumor as well as the distant metastasis.

Traditional therapeutic approaches such as surgery and chemotherapy are still considered to be effective in treating breast cancer [4]. Among all the chemotherapeutic drugs, docetaxel (DTX) is playing a significant role in treating metastatic breast cancer (MBC) and advanced breast cancer (ABC) [5]. As the first-line anti-tumor drug synthesized by the chemical substances in the yew tree, the anti-tumor effect of DTX is mainly achieved by destroying mitosis and cell proliferation [6]. Owning to its extensive antitumor efficiency, DTX is becoming one of the most effective chemotherapeutic agents in treating breast cancer [7]. However, chemotherapy agent usually induces undesirable effects as well as whole-body toxicity which have restricted the therapeutic efficiency greatly [8]. Besides, different clinical stages and various personal conditions reveal that a single therapeutic approach may not be efficient enough to meet all the expectations in breast cancer treatment. Hence, there is an urgent need to improve both the toxic side effect and treating effectiveness in the future application requirements.

Ultrasound was first explored in clinical diagnosis due to its extraordinary advantages such as radiationfree, noninvasiveness, and cost-effectiveness $[9,10]$. Despite the unique features mentioned above, it has 
also gained a lot of attention in therapeutic prospects. Sonodynamic therapy was initially realized by the cavitation and 'sonoporation effect' triggered by ultrasound. The generation of ROS during this process induces cytotoxicity toward cancer cells effectively, leading to rapid DNA damage and apoptosis of tumor cells [9]. Unlike the superficial employment of photodynamic therapy (PDT), SDT receives a more pleasant therapeutic outcome in treating deep-seated tumors by low intensity focused ultrasound (LIFU) which owns merits to prevent undesirable thermal injury during treatment [11-13]. As one of the essentials in SDT, sonosensitizers have been explored in various tumor therapies [14]. However, the single employment of the sonosensitizers has aroused instability as well as undesirable skin toxicity which have all limited the extensive SDT exploration.

The rapidly growing trend of nanotechnology combined with cancer treatment has been explored extensively, paving a feasible way in promoting multiple therapeutic strategies [15]. To realize effective tumor therapeutic applications, the delicately designed nanoparticles are set primarily to improve the toxic chemotherapy agent transportation efficiency via the enhanced permeability and retention (EPR) effect, enabling an increased accumulation in the tumor site as well $[16,17]$. With the integration of sonodynamic therapy and chemotherapy, the whole nanoplatform is of great value to revolutionize the traditional therapeutic efficiencies. Xu et al [18] demonstrated that due to SDT, an enhanced therapeutic result could be revealed through better-internalized chemotherapy drugs and an activation of mitochondria-targeted tumor cell apoptosis, which unveils the synergistic treatment pattern to be highly recommended in future utilization. Furthermore, the core-shell strategy can be administrated steadily without hurting normal tissues or cells, which is expected to minimize the side effect and toxicity once considered unavoidable [19-21].

Herein, with the inspirations mentioned above, we intent to exert the all-in-one nanoparticles (CPDP NPs) to establish a diagnostic and therapeutic system which is functionalized by its SDT capability, enhanced ultrasound imaging, and synergistic treating efficiency. Owning to the excellent safety and ideal metabolic stability as a desirable nanocarrier, PLGA has made it favorable in exploring various antitumor efficiencies [22, 23]. As a unique phase-shift material, PFP could significantly enhance ultrasound imaging by its phase-shift capability triggered by ultrasound. Ce6, a desirable second generation sonosensitizer, is capable of producing ROS through SDT treating strategy. After LIFU exposure, both chemotherapy and SDT will be realized to achieve a synergistic treating efficiency. Besides, the whole system is safe and stable with excellent biocompatibility. It is highlighted that lung metastasis as well as tumor growth have been remarkably inhibited both in vitro and in vivo. Hence, a productive treatment efficacy against breast malignant tumor and its distant metastasis might be considered as the potential treating strategy in further clinical application.

\section{Materials And Methods}

\section{Materials}


PLGA-COOH $₫$ Mw12000kDa『was purchased from Jinan Daigang Biomaterial Co., Ltd (Jinan, China). Perfluoropentane (PFP) and agarose were obtained from Sigma-Aldrich Co., Ltd(St. Louis, MO). Chlorin e6 (Ce6) was purchased from Melone Pharmaceutical Co., Ltd (Dalian, China). Cell Counting Kit-8 (CCK-8) cytotoxicity assay kit was obtained from Dojindo Molecular Technologies (Tokyo, Japan). 2',7'Dichlorodihydrofluorescein diacetate (H2DCFDA) and Calcein acetoxymethyl ester (Calcein-AM) were purchased from MedChemExpress Co., Ltd (NJ, USA). Propidium lodide (PI) was obtained from Solarbio Science \& Technology Co. Ltd (Beijing, China). Annexin V-FITC/PI were obtained from BD Biosciences (USA). Docetaxel (DTX) was purchased from MedChemExpress Co., Ltd (NJ, USA). All other reagents were analytical pure products without further purifications. Roswell Park Memorial Institute 1640 medium (DMEM), fetal bovine serum and tyrisin were purchased from Gibco (ThermoFisher Scientific, USA).

\section{Synthesis of CPDP NPS}

Ce6-PFP-DTX/PLGA nanoparticles (CPDP NPs) were prepared by a W/O/W double emulsion method according to the previous report [24]. Briefly, 2 mg Ce6 was firstly dissolved in $500 \mu$ methanol. Next, 50 mg PLGA-COOH was dissolved in $4 \mathrm{ml}$ dichloromethane and then the previous solution was simultaneously added into it. Then, $200 \mu \mathrm{l}$ PFP and Docetaxel (2 mg) were added to the above solution. In consequence, the mixture was triggered by an ultrasonic probe (Sonics \& Materials Inc., USA) to gain a first emulsion ( $5 \mathrm{~s}$ on and $5 \mathrm{~s}$ off, $3 \mathrm{~min}$ ). To acquire the second emulsion, $8 \mathrm{ml}$ of a poly (vinyl alcohol) (PVA) solution ( $\mathrm{w} / \mathrm{v}=4 \%$ ) was added into the above emulsion, using the same ultrasonic probe for 2 min. After adding $10 \mathrm{ml}$ 2\% isopropyl alcohol into the final emulsion, the solution was mixed under room temperature mechanically for at least $4 \mathrm{~h}$ to make dichloromethane volatilized totally. Finally, CPDP NPs were centrifugated for three times (12000 rpm, $5 \mathrm{~min}$ ) and then collected and stored at $4{ }^{\circ} \mathrm{C}$ for further use. The PDP NPs were prepared in the same way except for Ce6. All experimental processes were operated above ice and conducted strictly in the dark.

\section{Characterization of CDPD NPs}

The particle size and zeta potential of CPDP NPs and PDP NPs were realized by Malvern Zetasizer Nano instrument (Malvern, UK). The morphology was characterized by transmission electron microscopy (TEM) and optical microscopy. To evaluate the stability, CPDP NPs were dissolved in phosphate-buffered solution (PBS) and measured the sizes of 7 days respectively. Each sample was triplicate measured. The encapsulation efficiency of CPDP NPs were calculated through the following formula:

Encapsulation efficiency $(\%)=($ Weight of loading DTX or Ce6/Weight of total DTX or Ce6) $\times 100 \%$

\section{Drug releasing rate of CPDP NPS}

To evaluate the drug-releasing ability of Ce6 and DTX in CPDP NPs, two solutions with different $\mathrm{pH}$ (phosphate buffer solution, PBS: 7.4, acetate buffer solution, ABS: 5.6) were utilized to test the cumulative releasing efficiency. Briefly, CPDP NPs was firstly dispersed with $1 \mathrm{ml}$ PBS or ABS after the mixture was sealed into a dialysis bag (Mw:10000), the whole solution was then transferred into a glass 
bottle (total volume: $150 \mathrm{ml}$ ) in which $149 \mathrm{ml}$ PBS or ABS was added to keep the total solution volume at $150 \mathrm{ml}$. The glass bottle was then placed into a $37{ }^{\circ} \mathrm{C}$ constant temperature shaker, and at different periods $(0.5,1,2,4,8,12,24,48,72 \mathrm{~h})$, the solution was collected and immediately supplemented with the same volume of medium. Each group was repeated three times. Finally, the concentrations of Ce6 and DTX were measured by Synergy Hybrid Multi-Mode Read (BioTek, USA) at 403 and $229 \mathrm{~nm}$ respectively, and the drug-releasing rate at each time point was calculated.

\section{In vitro ultrasound imaging}

To investigate the ultrasonic capability of CPDP NPs, the emulsion $(1 \mathrm{mg} / \mathrm{ml})$ was firstly triggered by a low intensity focused ultrasound (LIFU) transducer equipment (Ronghai Ultrasonic Medical Engineering Research Center, Chongqing, China), and the conducting pattern was set as $50 \%$ duty cycle, $1 \mathrm{~s}$ pulse duration under different intensities $\left(1-2 \mathrm{~W} / \mathrm{cm}^{2}\right)$ for different duration time. For ultrasound imaging, irradiated CPDP NPs were added into the previous prepared agarose model respectively, using Philips EPIQ5 ultrasound diagnostic instrument (probe frequency:12Mhz, MI:0.06) to observe both 2D and CEUS imaging of CPDP NPs. Meanwhile, ImageJ software was applied to analyze the gray scale value of each group.

\section{Cell culture and in vitro ROS generation of CPDP NPs by LIFU irradiation}

The murine breast cancer cell line 4T1 was obtained from Shanghai cell bank of the Chinese Academy of Sciences (Shanghai, China) and incubated in RPMI 1640 medium mixed with 10\% FBS and 1\% streptomycin/penicillin at $37{ }^{\circ} \mathrm{C}$ in a $5 \% \mathrm{CO}_{2}$ humidified incubator.

$4 \mathrm{~T} 1$ cells were incubated as previous condition at a density of $1 \times 10^{4}$ cells per dish and separated into the following 5 groups: Control, CPDP NPs, LIFU, Ce6 + LIFU, CPDP NPs + LIFU. After $24 \mathrm{~h}$ conventional culture, the medium was replaced by CPDP NPs $(200 \mu \mathrm{l}, 0.8 \mathrm{mg} / \mathrm{ml})$ or Ce6 solution $(200 \mu \mathrm{l})$ respectively and the cells were co-incubated for another $3 \mathrm{~h}$. Then LIFU irradiation $\left(2 \mathrm{~W} / \mathrm{cm}^{2}, 120 \mathrm{~s}\right)$ was conducted respectively according to different groups. After co-incubation and LIFU treatment, $100 \mu$ liluted DCFHDA solution was added and each group was cultured in the previous incubator for $15 \mathrm{~min}$. A confocal laser scanning microscope (CLSM) was used to confirm the result of reactive oxygen species production and the corresponding fluorescence intensities were measured by ImageJ software.

\section{In vitro cytotoxicity and concerted treatment capability of CPDP NPs}

A CCK-8 assay was applied to assess the cytotoxicity of CPDP NPs. Briefly, 4T1 murine breast cancer cells were incubated in a 96-well plate, with a density of $1 \times 10^{4}$ per well for $24 \mathrm{~h}$. Then CPDP NPs were diluted with serum-free RPMI 1640 medium at various concentrations $(0,0.2,0.4,0.6,0.8 \mathrm{mg} / \mathrm{ml}, \mathrm{n}=3)$, with or without LIFU irradiation ( $2 \mathrm{~W} / \mathrm{cm}^{2}, 120 \mathrm{~s}$ ). After another $6 \mathrm{~h}$ of cocultured process, the cell viability of $4 \mathrm{~T} 1$ cells was performed. 
To evaluate cell apoptosis efficiency of concerted treatment, 4T1 cells were cocultured as previous for 24h and then separated into five following groups: (1) Control (without any treatments), (2) LIFU (only with LIFU exposure at $2 \mathrm{~W} / \mathrm{cm}^{2}$ ), (3) CPDP NPs (only with CPDP NPs solution at $0.8 \mathrm{mg} / \mathrm{ml}$ ), (4) PDP NPs + LIFU and (5) CPDP NPs + LIFU. After various nanoparticles coincubation $(200 \mu \mathrm{l})$ and LIFU exposure, each group was treated with Annexin V $(5 \mu \mathrm{l})$ and Propidium lodide $(5 \mu \mathrm{l})$ double staining for 20 min and analyzed through a flow cytometry protocol.

\section{In vitro inhibition of cell metastasis}

To investigate the inhibition of cell metastatic ability, wound healing assay and transwell assay were designed. For wound healing assay, 4T1 cells were conventional cultured as previous in the 6-well plate. After cell growth to an $80 \%$ confluency, a pipette tip (10 $\mu$ l) was applied to conduct a manmade scratch along the center of the 6 -well plate. Then cells were treated in the same groups mentioned above. After a continuously coincubation for 24 hours, the cells were washed with PBS 3 times and observed under optical microscopy (Olympus, Japan).

For transwell assay, the top compartment of the transwell chamber (Corning, San Diego, USA) was primarily applied to imitate the extracellular matrix in vivo. $4 \mathrm{~T} 1$ cells at a density of $1 \times 10^{5}$ cells per well were seeded into the upper chamber in a serum-free RPMI 1640 medium, while the bottom compartment was filled with a complete culture medium mixed with $10 \%$ FBS. Then cells were separated as the same as above groups and treated for $24 \mathrm{~h}$ respectively. After that, cells in the bottom surface were fixed with paraformaldehyde and stained with crystal violet. The results were observed with light microscopy (Olympus, Japan).

\section{In vivo synergistic therapeutic efficiency of CPDP NPs}

Healthy female BALB/c mice (4 weeks) and Kunming mice (4 weeks) were obtained from Ningxia Medical University Laboratory Animal Center. All animal experiments were conducted under the guideline approved by the Animal Welfare Ethics Review Committee of Ningxia Medical University. To establish the mice tumor-bearing model, BALB/c mice were inoculated with $4 \mathrm{~T} 1$ breast cancer cells $\left(1 \times 10^{7} / \mathrm{ml}\right)$ at the right flank. The size of the tumor was recorded every two days, and the volume of the tumor was calculated by the formula as: Volume $=1 / 2 \mathrm{x}$ Length $\mathrm{x}$ Width ${ }^{2}$. Tumor size and mice body weight were recorded every 2 days while the pictures of tumor growth were recorded every 3 days. When tumor volume reached $60-80 \mathrm{~mm}^{3}$, mice with similar tumor size were randomly divided into the same 5 groups: Control, LIFU, CPDP NPs, PDP + LIFU, and CPDP NPs + LIFU $(n=3)$. Each group was intravenously injected with various NPs $(200 \mu \mathrm{l})$ via tail vein except for Control group (200 $\mu \mathrm{l}$ PBS instead). Twenty-four hours later, the tumor site was exposed with LIFU irradiation $\left(2 \mathrm{~W} / \mathrm{cm}^{2}\right)$ for $120 \mathrm{~s}$. The whole SDT administration was repeated every 3 days and lasted for 18 days. The body weight and tumor volume of the mice were measured and calculated. After the treatment, mice were sacrificed and tumor tissues were sent to H\&E, TUNEL, and PCNA for further histological analysis. 


\section{In vivo ultrasound imaging}

Tumor-bearing BALB/c mice were injected with CPDP NPs $(200 \mu \mathrm{l}, 1 \mathrm{mg} / \mathrm{ml})$ through the tail vein intravenously. After 24 hours, tumor sites of the mice were conducted with LIFU $\left(2 \mathrm{~W} / \mathrm{cm}^{2}, 120 \mathrm{~s}\right)$, then the 2D and CEUS imaging were acquired through the Philips EPIQ5 ultrasound diagnostic instrument mentioned previously. The gray scale analysis was measured by ImageJ software.

\section{Biosafety of CPDP NPs in vivo}

To investigate the biosafety of CPDP nanoparticles in vivo, healthy female Kunming mice $(n=3)$ were separated into the following 4 groups: Control, $5 \mathrm{mg} / \mathrm{ml}, 10 \mathrm{mg} / \mathrm{ml}$, and $20 \mathrm{mg} / \mathrm{ml}$. The CPDP NPs (200 $\mu \mathrm{l})$ were injected through the mice tail vein, then the mice were free access to food and water without any further administration. The bodyweight of mice was measured every 2 days. After 30 days, mice were sacrificed and the blood samples were collected for blood cell and biochemistry analysis. The major organs (heart, liver, spleen, lung, and kidney) were collected and investigated for H\&E staining respectively.

\section{In vivo inhibition of lung metastasis}

To evaluate the lung metastasis inhibition of each group, the whole process was conducted by observing the number of metastatic nodules in the lung as well as an H\&E staining histology assessment. After all the mice were euthanized, the lung tissues were removed and fixed, then photographs of cancer nodules were taken and lung tissues were further analyzed with H\&E staining.

\section{Statistical analysis}

Measurement data were all performed 3 times and expressed as mean \pm standard deviation (SD) and analyzed by one-way ANOVA analysis or a standard Student's t-test through the SPSS software (version:19.0), while $p$ value $<0.05$ was considered to be statistically significant.

\section{Results}

\section{Characterization of CPDP NPs and drug-releasing efficiency}

A double emulsion method was applied in fabricating CPDP NPs which encapsulated both phase-shift material PFP, sonosensitizer Ce6, and chemo-drug DTX simultaneously. When dispersed into PBS or deionized water, the solution presented a light gray appearance. CPDP NPs exhibited a homogeneous spherical shape and a clear core-shell structure, whether being observed via optical microscopy or transmission electron microscopy (Figure $1 \mathrm{a}, \mathrm{b}$ ). The mean diameters of CPDP NPs and PDP NPs were $249.5 \pm 77.46 \mathrm{~nm}$ and $246.6 \pm 81.01 \mathrm{~nm}$, and the average surface zeta potentials were $-18.47 \pm 0.5508 \mathrm{mV}$ and $-3.987 \pm 0.6573 \mathrm{mV}$, respectively (Figure 1c, d). The size of CPDP NPs guaranteed that it could be accumulated into the tumor site passively through the EPR effect [25]. In addition, the negative zeta potential of CPDP NPs indicated a lower plasma protein adsorption, verifying the relative stability of 
nanoparticles. The particle size distributions were maintained between a range of 249.5 and $385.1 \mathrm{~nm}$ in 7 days (Figure 1e), demonstrating the relative steadiness of CPDP NPs. According to the standard curve, the encapsulation efficiency of DTX and Ce6 were $83.84 \pm 1.39 \%$ and $60.54 \pm 3.79 \%$, respectively.

As drug-releasing efficiency of Ce6 and DTX from CPDP NPs indicated in Figure 1g, a nearly 2 -fold increase of DTX releasing index was recorded at pH 5.5 compared with nanoparticles dissolved in $\mathrm{pH} 7.4$, which indicated the reasonable drug releasing rate of DTX could be achieved effectively in an acid tumor microenvironment. Interestingly, the result in Figure $1 \mathrm{f}$ indicated that even at the condition of $\mathrm{pH} 5.5$, the releasing efficiency of Ce6 was still around $20 \%$. This minor releasing rate demonstrated that Ce6 could be effectively protected in PLGA so that substantial ROS generation through LIFU stimulation could be reached to get a more convinced SDT efficiency. The above results in all exhibited the delicate designed CPDP NPs can exert a steady and timely chemotherapeutic drug release in the acid tumor environment, and also fundamentally desirable to be prepared for SDT.

\section{In vitro ultrasound imaging}

It is highlighted that PFP owns excellent phase-shift ability. The liquid to gas transformation not only helps nanoparticles aggregate within the tumor site but also entitles its capability to enhance the efficiency of ultrasound imaging [26]. To demonstrate that, LIFU irradiation was applied as a trigger to induce the phase transformation of PFP, namely, the acoustic droplet vaporization (ADV) effect [27]. The results showed that the gray scale intensities were kept at a relatively low level before LIFU irradiation, while after the intensity and irradiating time of LIFU increased, the tendency of enhanced ultrasound imaging was revealed both in 2D and CEUS (Figure 2a). The acoustic analysis of ImageJ further convinced the result by elevated gray scale value (Figure 2b, c), which was consistent with the imaging findings. It should be noted that the most significant result of 2D and CEUS gained when LIFU intensity achieved $2 \mathrm{~W} / \mathrm{cm}^{2}$ and lasted for $120 \mathrm{~s}$. The results above demonstrated that PFP was successfully encapsulated in CPDP NPs, and ultrasound imaging capability had been significantly promoted under higher intensity as well as longer time of LIFU administration.

\section{In vitro ROS generation of CPDP NPs by LIFU irradiation}

The main strategy of sonodynamic therapy (SDT) is the generation of ROS-a series of single-electron reduction products-to induce cancer cell apoptosis and inhibit cell proliferation [28]. It is highlighted that when exposed to ultrasound, the sonosensitizer is prone to trigger ROS production, meanwhile, a considerable amount of energy will be released during the whole process [28,29]. Given that both ultrasound and sonosensitizer are dispensable elements to promote SDT, hence, the intracellular ROS generation was designed and analyzed to investigate the differences between divided groups. According to Figure 3a, the amount of ROS generated by the free Ce6 plus LIFU irradiation group was negligible, which may because the rapid metabolism of free Ce6 leads to an unsatisfactory ROS production. On the contrary, the strongest fluorescent intensity was revealed by CPDP NPs + LIFU group. It was assumed that the encapsulated Ce6 was well protected and thus had been kept away from being metabolized. As a 
consequence, after LIFU stimulation, Ce6 was released to produce abundant ROS. Comparatively, there were no significant fluorescent signals found in other groups (Figure 3b).

\section{In vitro cytotoxicity and SDT capability of CPDP NPs}

Cell Counting Kit-8 (CCK-8) assay was introduced to test the in vitro cytotoxicity of CPDP NPs. In this respect, different groups were designed with or without LIFU irradiation at different concentrations. The results indicated that after a $24 \mathrm{~h}$ co-incubation without LIFU exposure, there was no obvious effect of the survival rate of CPDP NPs even at the highest concentration $(0.8 \mathrm{mg} / \mathrm{ml})$, demonstrating the desirable biosafety of CPDP NPs (Figure 4a). By contrast, it showed that there was a striking decrease of cell viability after LIFU irradiation, showing the combination of CPDP NPs and LIFU has remarkably triggered 4T1 cell death which was consistent with the in vitro ROS generation.

To further evaluate SDT efficacy, a flow cytometry assay was introduced. As the results shown in Figure $4 \mathrm{~b}$ and $4 \mathrm{c}$, the index of cell necrosis and apoptosis was highest observed in CPDP NPs + LIFU group, while other groups showed no obvious and necrosis and apoptosis. Notably, the necrosis and apoptosis rate of CPDP NPs + LIFU group was 3-fold higher than that of CPDP NPs only group, which ensured the significant tumor cell death efficiency of SDT from another respect. Intriguingly, compared with PDP NPs + LIFU group, cell necrosis and apoptosis rate in CPDP NPs + LIFU group was significantly increased, exhibiting the synergistic therapy efficiency of SDT and chemotherapy.

\section{In vitro inhibition of cell metastasis}

The invasive and migration capability of tumor cells are indispensable in tumor progression [30]. As shown in Figure 5 a, the closure between the physical gap of CPDP NPs + LIFU group was significantly smaller than other groups, indicating a relatively slower speed of migrating efficiency. According to the Image J software analysis (Figure 5b), the migration rate of CPDP NPs + LIFU group was also remarkably reduced compared with other groups.

Similarly in the transwell assay, compared with the swift migration speed of other groups, CPDP NPs + LIFU group revealed a significant reduction of cell number (Figure 5c), which demonstrated an excellent anti-migration capability of the synergistic therapy. Specifically, with the absence of SDT (CPDP NPs only and LIFU only group), the number of tumor cells was mildly decreased (Figure $5 c$, d). On the whole, due to the combination of SDT as well as chemotherapy, metastasis of $4 \mathrm{~T} 1$ cells has been remarkably inhibited in vitro.

\section{In vivo synergistic therapeutic efficiency of CPDP NPs}

Seeing from Figure $6 a$ and $6 \mathrm{~b}$, the tumor volume of CPDP NPs+ LIFU group was significantly smaller after 18 days of treatment than that of other groups, which may attribute to the effectiveness of ROS originated from SDT treatment as well as chemotherapy to exert a valid synergistic therapy efficiency. Similarly, photos of mice bearing tumors (Figure 6a) also showed the same trend, verifying the cooperative treating efficacy of CPDP NPs triggered by LIFU exposure. Furthermore, there was no obvious 
weight reduction of mice between different groups (Figure 6c). The results above all indicated a much higher inhibition rate of CPDP NPs + LIFU group, revealing the synergistic therapy could significantly prevent tumor growth.

To further testify the therapeutic results of all groups, both H\&E, TUNEL, and PCNA staining were utilized (Figure $6 \mathrm{~d}$ ). The proliferate rate of PCNA in CPDP NPs + LIFU group was only $20.50 \%$, which was 4 -folds lower than Control group, 3-folds lower than LIFU and CPDP NPs only group and 2-folds lower than PDP + LIFU group respectively, demonstrating a significant anti-tumor proliferation rate (Figure 6e). As it was shown in Figure $6 \mathrm{~d}$ and $6 \mathrm{f}$, the TUNEL results indicated CPDP NPs + LIFU group exhibited an obvious apoptosis index of $72.86 \%$, which was much higher than Control (9.66\%), LIFU (12.86\%), CPDP NPs (19.59\%), and PDP NPs + LIFU (37.06\%) group. The results above all demonstrated the effectiveness of synergistic therapy exerted in vivo, which was also proved consistent with the previous in vitro results.

\section{In vivo ultrasound imaging}

Since PFP was encapsulated in CPDP NPs, it is also necessary to evaluate the characteristic ultrasound imaging capability in vivo. After the injection via the tail vein of CPDP NPs, LIFU was then applied to the tumor site to acquire both 2D and CEUS imaging (Figure 7a). The clear graphic difference between the two groups indicated that after LIFU irradiation, the corresponding intensity of CPDP NPs was elevated obviously compared with the pre-irradiation group. Further data of the average echo intensity also confirmed this result which was also consistent with the in vitro imaging result previously (Figure 7b, c).

\section{Biosafety of CPDP NPs in vivo}

Despite the effective therapeutic outcome, it is of great importance to explore the biosafety of the novel established nanoparticles as well. On behalf of the safe distribution of CPDP NPs in vivo, the metabolic safety was conducted. The results showed that instead of apparent body weight loss, the mice body weight elevated gradually in all the groups of mice (Figure 8a), which indicated a negligible negative influence of CPDP NPs. In addition, as various organs as well as the blood samples exhibited in Figure $8 b$, no significant changes were observed in blood cell and biochemistry analysis index as well as H\&E staining (Figure 8c) among different treating groups, suggesting the excellent biosafety of CPDP NPs in vivo.

\section{In vivo inhibition of lung metastasis}

It is well established that the lung is the main target organ of breast cancer distant metastasis [31]. In order to evaluate the suppression efficiency of metastasis, lung tissues of mice were utilized for investigation. As seen from Figure 9a and 9b, compared with Control, LIFU, CPDP NPs, and PDP + LIFU group, the CPDP NPs + LIFU group still exhibited the most remarkable decrease in the number of lung nodules, which suggested its desirable lung metastatic inhibition efficiency. The result of H\&E staining also further indicated a similar trend of decreasing in pulmonary lesions (Figure 9c), which in all demonstrated the effectiveness in eliminating lung metastasis by this synergistic therapy strategy. 


\section{Discussion}

It has been greatly acknowledged that the metastasis of breast cancer will extensively influence its poor prognosis [3,32]. SDT may serve as the desirable approach through its high efficiency and deep penetration capability has been extensively acknowledged $[11,33]$. Admittedly, single-use of SDT may still be less sufficient in further clinical cancer exploration since certain limitations exist in the single application of sonosensitizers. The development of nanotechnology combined with clinical medicine has been promoted significantly in recent years, owning inspiring merits such as negligible toxicity, none invasiveness, and excellent biocompatibility. Hence, many efforts have been made in exploring multifunctional therapeutic strategies to realize antitumor efficiency [29].

The biosafety is the priority of nano agents. As a widely accepted material approved by the Food and Drug Administration (FDA) certification, it is highlighted that PLGA could be performed as a desirable carrier in application [34,35]. Based on its advantages, we established a nano-system to realize multifunctional therapy efficiency, exploiting PLGA as the outer structure to encapsulate sonosensitizer Ce6, phase-shift material PFP and chemotherapeutic agent DTX. The CPDP nanoparticles (CPDP NPs) were primarily observed by the core-shell structure and appropriate size so that a desirable aggregation through the EPR effect could be achieved. The cell viability of CPDP NPs has been proved to be above $80 \%$ after 24 hours coincubation, indicating the well safety of this nanoplatform. Besides, the phase transformation of encapsulated PFP has also guaranteed CPDP NPs as a contrast enhanced agent when activated by LIFU. The enhanced ultrasound imaging capability will not only ensure the ideal therapeutic window but also promote the promising future for CPDP NPs to realize an integration of accurate diagnosis and precise treatment.

The key strategy in SDT is the generation of ROS [28]. Compared with the single employment of Ce6, the encapsulated Ce6 in PLGA exerted a desirable protection which could be verified by the ROS result. In our study, there was only a negligible amount of ROS generated by single-use of Ce6, while nanoparticle encapsulated Ce6 produced a considerable amount of ROS which was further proved by SDT research. The result indicated that ROS generation was preserved by the encapsulated Ce6 in CPDP NPs which could lay a firm foundation for later tumor inhibition. Besides, the drug releasing rate showed even at $\mathrm{pH}$ 5.5, little Ce6 was released from nanoparticles, demonstrating a well protection of sonosensitizer by PLGA which was further proved by the ROS production and SDT outcome with high efficiency. Current researches have suggested that using chemotherapy alone may not significantly reverse tumor progression [29]. In this study, the nanoplatform we designed demonstrated strong evidence that compared with the single employment of chemotherapy, the synergistic treatment has remarkably elevated therapeutic efficiency both in vitro and in vivo. Since LIFU stimulation optimized the therapeutic strategy, an increased cell apoptosis rate was remarkably elevated. It is worth noted that lung metastasis could be significantly inhibited both at tumor cell level as well as inoculated mice model, which is consistent with previous reports [37-38].

\section{Conclusion}


In conclusion, we designed and prepared CPDP NPs which were proved by productively reducing tumor proliferation as well as inhibiting lung metastasis caused by breast cancer. The CPDP NPs plus LIFU irradiation could remarkably eliminated breast tumor progression and its lung metastasis. With an enhanced imaging capability, this nanoplatform was also considered to be a promising contrast enhanced agent in clinical. It is highlighted that this novel synergistic strategy combined with LIFU might be considered as an effective treating application with extensive potential in reversing the poor outcome of metastatic breast cancer in future applications.

\section{Abbreviations}

LIFU: Low intensity focused ultrasound; SDT: Sonodynamic therapy; PFP: perfluoropentane; DCFH-DA: Dichlorodihydrofluorescein diacetate, Chlorin e6: Ce6; DTX: docetaxel; FBS:Fetal bovine serum; CCK-8: Cell counting kit-8

\section{Declarations}

\section{Authors' Contributions}

QZ, WW, and CM conceived the idea and designed the experiment. QZ conducted the experiment and prepared the manuscript. QZ, WW, HS, HT, YW, LM, GY, RC and CM revised the manuscript. All authors approved the manuscript.

\section{Conflict of interest}

The authors declare that there is not any conflict of interest regarding this paper.

\section{Funding}

This paper was supported by the National Science Foundation of China (Grant no. 81360225, and 81960317)

\section{Acknowledgements}

We gratefully acknowledge the assistance of $Y Z$ for kindly assisting with the experimental devices. We also thank YF for revising the manuscript.

\section{Author details}

${ }^{1}$ Department of Ultrasound, General Hospital of Ningxia Medical University, Yinchuan, China.

${ }^{2}$ School of Clinical Medicine, Ningxia Medical University, Yinchuan, China.

\section{References}


1. Bray F et al.(2018) Global cancer statistics 2018: GLOBOCAN estimates of incidence and mortality worldwide for 36 cancers in 185 countries. CA Cancer J Clin, 68(6), 394-424.

2. Redig AJ et al (2013) Breast cancer as a systemic disease: a view of metastasis. J Intern Med, 274(2), 113-26.

3. Li Z et al.(2016) Emerging therapeutic targets in metastatic progression: A focus on breast cancer. Pharmacol Ther, 161, 79-96.

4. Wang W et al.(2017) Anticancer Effects of Resveratrol-Loaded Solid Lipid Nanoparticles on Human Breast Cancer Cells. Molecules, 22(11), undefined.

5. Fiocchetti M et al (2020) Extracellular Neuroglobin as a Stress-Induced Factor Activating PreAdaptation Mechanisms against Oxidative Stress and Chemotherapy-Induced Cell Death in Breast Cancer. Cancers (Basel), 12(9).

6. Varshosaz J et al.(2015) Folated synperonic-cholesteryl hemisuccinate polymeric micelles for the targeted delivery of docetaxel in melanoma. Biomed Res Int, 2015, 746093.

7. Wang M et al.(2020) Development and Evaluation of Docetaxel-Phospholipid Complex Loaded SelfMicroemulsifying Drug Delivery System: Optimization and In Vitro/Ex Vivo Studies. Pharmaceutics, 12(6).

8. Alshaker H et al.(2017) New FTY720-docetaxel nanoparticle therapy overcomes FTY720-induced lymphopenia and inhibits metastatic breast tumour growth. Breast Cancer Res Treat, 165(3), 531543.

9. Xu M et al.(2021) Sonodynamic therapy-derived multimodal synergistic cancer therapy. Cancer Lett, 497, 229-242.

10. Liang S et al.(2020) Recent Advances in Nanomaterial-Assisted Combinational Sonodynamic Cancer Therapy. Adv Mater, 32(47), e2003214.

11. An J et al.(2020) ROS-augmented and tumor-microenvironment responsive biodegradable nanoplatform for enhancing chemo-sonodynamic therapy. Biomaterials, 234, 119761.

12. Wang $X$ et al.(2018) Enhanced drug delivery using sonoactivatable liposomes with membraneembedded porphyrins. J Control Release, 286(undefined), 358-368.

13. Zhao X et al.(2020) A Cleverly Designed Novel Lipid Nanosystem: Targeted Retention, Controlled Visual Drug Release, and Cascade Amplification Therapy for Mammary Carcinoma in vitro. Int $\mathrm{J}$ Nanomedicine, 15(undefined), 3953-3964.

14. Son S et al.(2020) Multifunctional sonosensitizers in sonodynamic cancer therapy. Chem Soc Rev, 49(11), 3244-3261.

15. Zhang X et al.(2019) Multifunctional Polyethylene Glycol (PEG)-Poly (Lactic-Co-Glycolic Acid) (PLGA)-Based Nanoparticles Loading Doxorubicin and Tetrahydrocurcumin for Combined Chemoradiotherapy of Glioma. Med Sci Monit, 25(undefined), 9737-9751. doi:10.12659/MSM.918899 
16. Wang $Y$ et al.(2018) Multifunctional Cargo-Free Nanomedicine for Cancer Therapy. Int J Mol Sci, 19(10).

17. Yang M et al (2018) A Modular Coassembly Approach to All-In-One Multifunctional Nanoplatform for Synergistic Codelivery of Doxorubicin and Curcumin. Nanomaterials (Basel), 8(3).

18. Xu J et al.(2011) Sonodynamic action of pyropheophorbide-a methyl ester induces mitochondrial damage in liver cancer cells. Ultrasonics, 51(4), 480-4.

19. Chen J et al.(2017) Theranostic Multilayer Capsules for Ultrasound Imaging and Guided Drug Delivery. ACS Nano, 11(3), 3135-3146.

20. Rapoport $\mathrm{N}$ et al.(2007) Multifunctional nanoparticles for combining ultrasonic tumor imaging and targeted chemotherapy. J Natl Cancer Inst, 99(14), 1095-106.

21. Wei W et al.(2020) in situResearch progress in the application of hydrogel system in tumor treatment. Drug Deliv, 27(1), 460-468.

22. Shen $X$ et al.(2020) PLGA-Based Drug Delivery Systems for Remotely Triggered Cancer Therapeutic and Diagnostic Applications. Front Bioeng Biotechnol, 8, 381.

23. Zhao Z et al.(2017) A nanoparticle-based nicotine vaccine and the influence of particle size on its immunogenicity and efficacy. Nanomedicine, 13(2), 443-454.

24. Cao Y et al.(2018) Drug Release from Phase-Changeable Nanodroplets Triggered by Low-Intensity Focused Ultrasound. Theranostics, 8(5), 1327-1339.

25. García-Pinel B et al.(2019) Lipid-Based Nanoparticles: Application and Recent Advances in Cancer Treatment. Nanomaterials (Basel), 9(4).

26. Yang M et al.(2020) Fabrication of doxorubicin-gated mesoporous polydopamine nanoplatforms for multimode imaging-guided synergistic chemophotothermal therapy of tumors. Drug Deliv, 27(1), 367377.

27. Liu J et al.(2018) Low-intensity focused ultrasound (LIFU)-activated nanodroplets as a theranostic agent for noninvasive cancer molecular imaging and drug delivery. Biomater Sci, 6(11), 2838-2849.

28. Zhang L et al.(2019) Mitochondria-Targeted and Ultrasound-Activated Nanodroplets for Enhanced Deep-Penetration Sonodynamic Cancer Therapy. ACS Appl Mater Interfaces, 11(9), 9355-9366.

29. Pan X et al.(2020) MOF-Derived Double-Layer Hollow Nanoparticles with Oxygen Generation Ability for Multimodal Imaging-Guided Sonodynamic Therapy. Angew Chem Int Ed Engl, 59(32), 1355713561.

30. Truffi M et al.(2019) Nano-Strategies to Target Breast Cancer-Associated Fibroblasts: Rearranging the Tumor Microenvironment to Achieve Antitumor Efficacy. Int J Mol Sci, 20(6).

31. Xie H et al.(2017) Tumor microenvironment: driving forces and potential therapeutic targets for breast cancer metastasis. Chin J Cancer, 36(1), 36.

32. Liang Y et al.(2020) Metastatic heterogeneity of breast cancer: Molecular mechanism and potential therapeutic targets. Semin Cancer Biol, 60(undefined), 14-27. 
33. Zheng X et al.(2019) Biodegradable Natural Product-Based Nanoparticles for Near-Infrared Fluorescence Imaging-Guided Sonodynamic Therapy. ACS Appl Mater Interfaces, 11(20), 1817818185.

34. Manaspon C et al.(2017) Increasing Distribution of Drugs Released from In Situ Forming PLGA Implants Using Therapeutic Ultrasound. Ann Biomed Eng, 45(12), 2879-2887. doi:10.1007/s10439017-1926-1

35. Sindeeva 0 et al.(2018) Effect of a Controlled Release of Epinephrine Hydrochloride from PLGA Microchamber Array: In Vivo Studies. ACS Appl Mater Interfaces, 10(44), 37855-37864.

36. Wang $P$ et al.(2015) Anti-metastatic and pro-apoptotic effects elicited by combination photodynamic therapy with sonodynamic therapy on breast cancer both in vitro and in vivo. Ultrason Sonochem, 23(undefined), 116-27.

37. Zhang D et al.(2020) Ultrasound-Driven Biomimetic Nanosystem Suppresses Tumor Growth and Metastasis through Sonodynamic Therapy, CO Therapy, and Indoleamine 2,3-Dioxygenase Inhibition. ACS Nano, 14(7), 8985-8999.

38. Zhao H et al.(2020) Biomimetic Decoy Inhibits Tumor Growth and Lung Metastasis by Reversing the Drawbacks of Sonodynamic Therapy. Adv Healthc Mater, 9(1), e1901335.

\section{Figures}
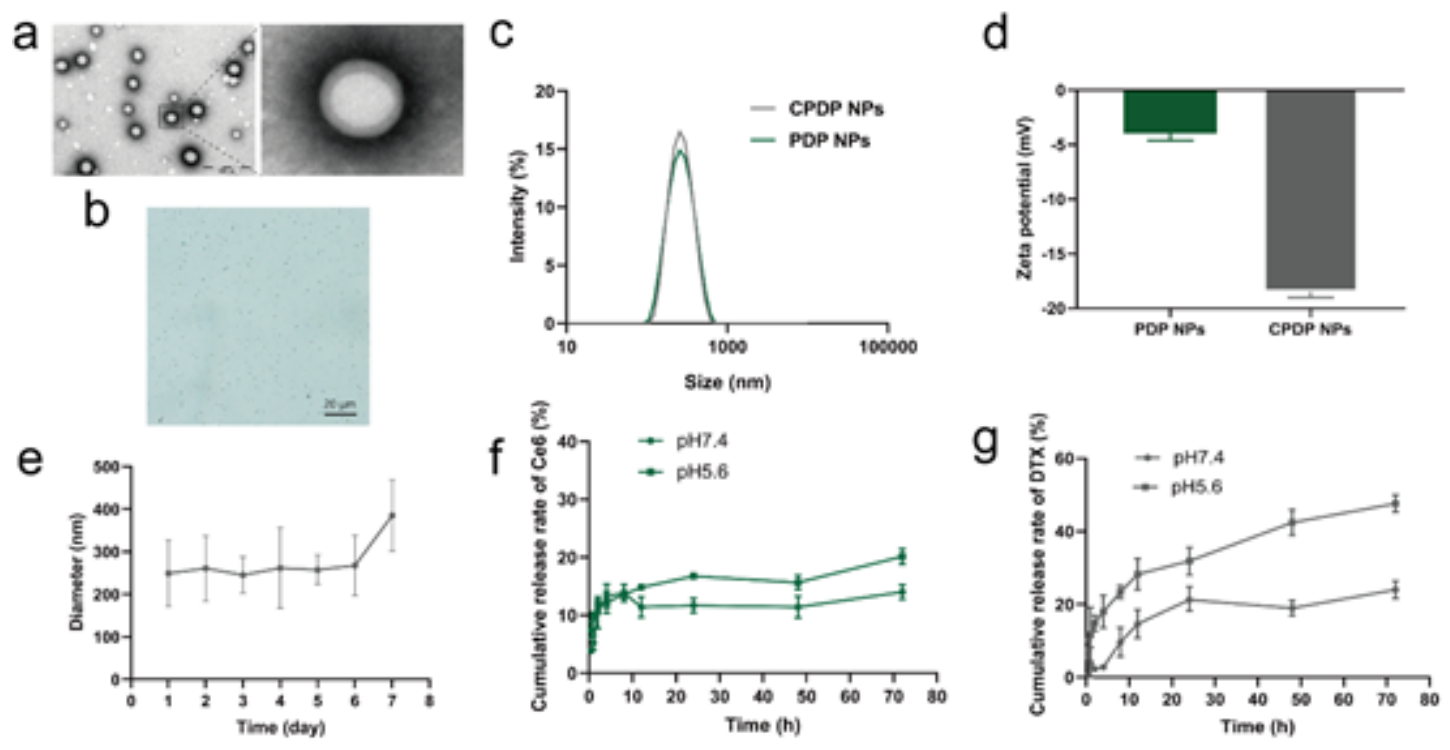

\section{Figure 1}

(a) TEM (scale bar: $500 \mathrm{~nm}$ ) and (b) Light microscope image of CPDP NPs (scale bar:20 $\mu \mathrm{m}$ ). (c) Size distribution and (d) Zeta potential of PDP NPs and CPDP NPs. (e) The size distribution of CPDP NPs within 7 days. (f) The releasing rate of $\mathrm{Ce} 6$ and $(\mathrm{g})$ The releasing rate of DTX under different conditions (pH7.4 and pH5.6, n=3). 
a
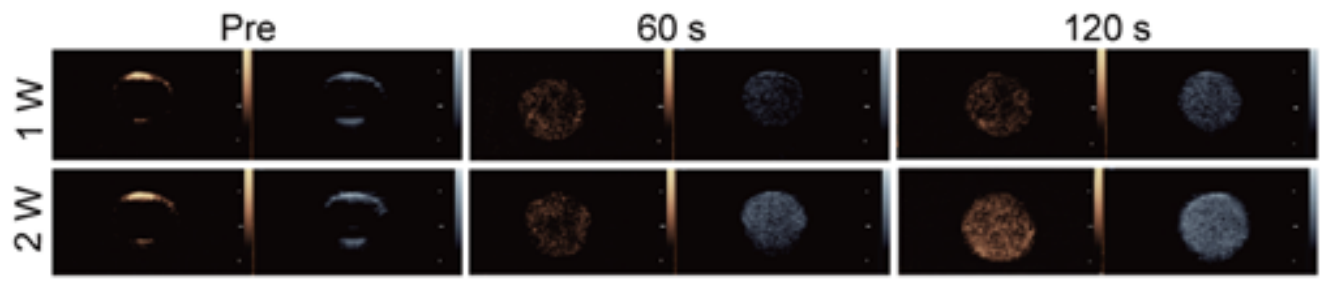

b
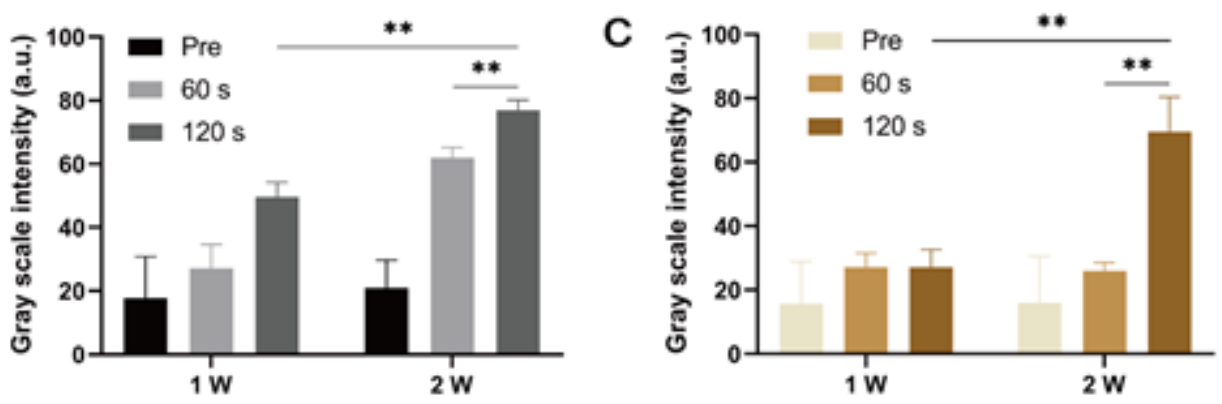

Figure 2

(a) Ultrasound images of both 2D and CEUS under different LIFU intensity and duration time. (b) The corresponding gray scale intensity at different intensities and time $(* * p<0.01, n=3)$.

a
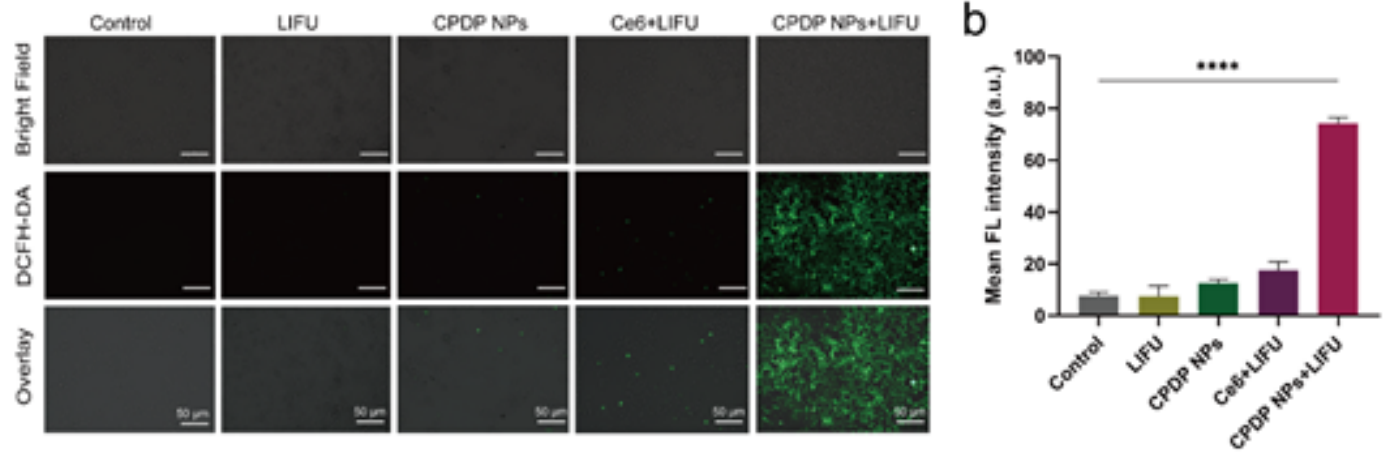

Figure 3

(a) CLSM images of ROS generation with various treatments and (b) The corresponding FL intensity analysis ( $\left.{ }^{* \star \star *} p<0.001, n=3\right)$. The scale bars are $50 \mu \mathrm{m}$. 
a

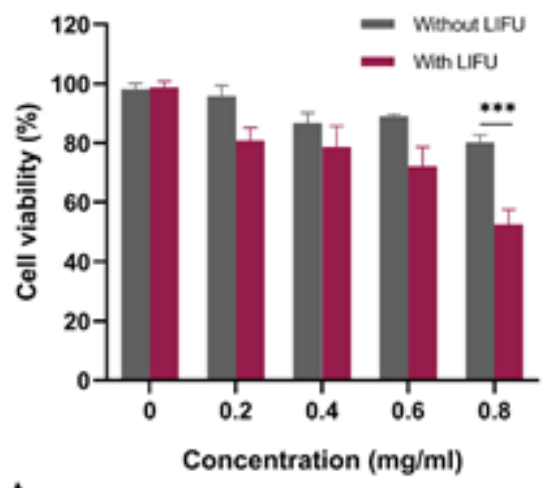

C

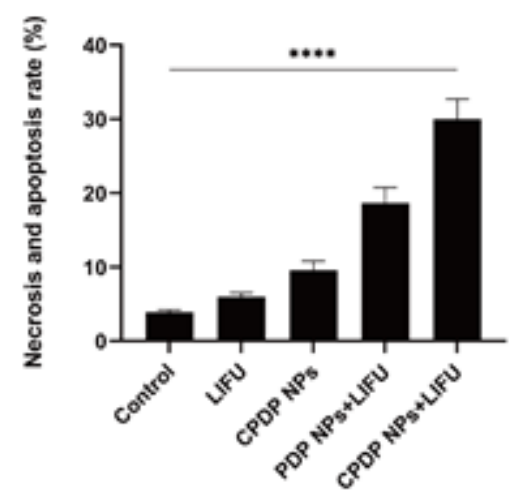

b

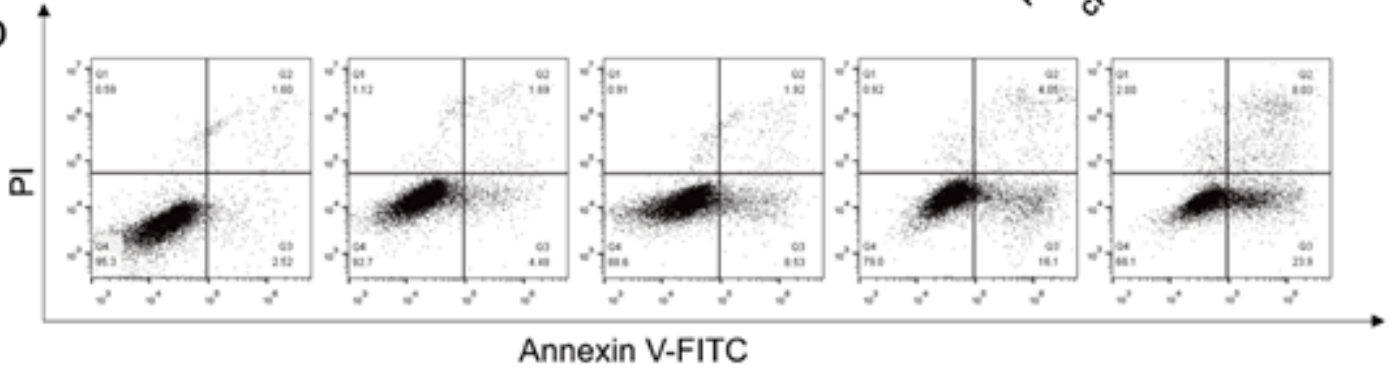

\section{Figure 4}

(a) Relative cell viability with or without LIFU irradiation under different CPDP NPs concentrations. (b) 4T1 tumor cell apoptosis and necrosis by flow cytometry assay and (c) The data of corresponding necrosis and apoptosis rate analysis ( ${ }^{* * *} p<0.001$, $* * * p<0.01, n=3$ ).

a

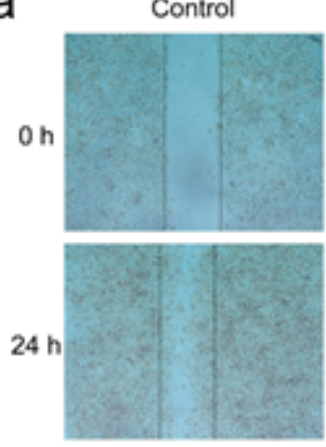

C

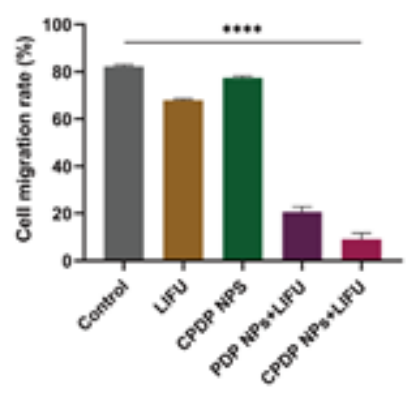

b

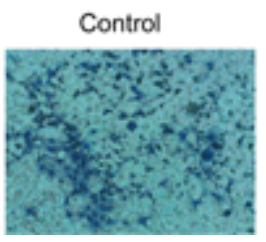

LIFU
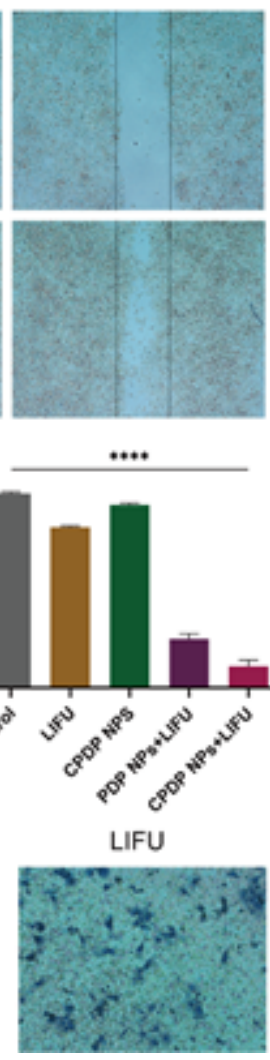

CPDP NPS
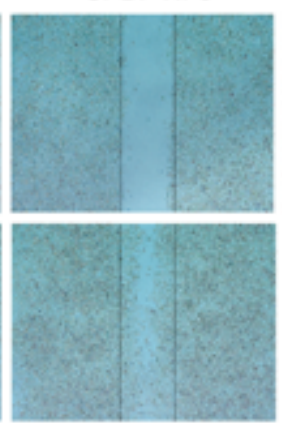

d

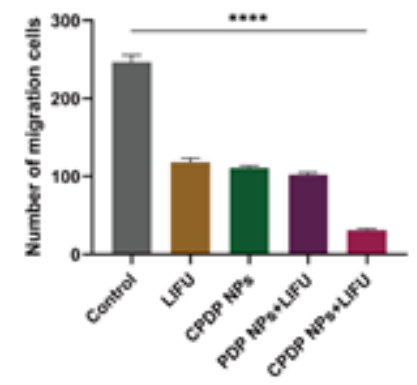

CPDP NPS

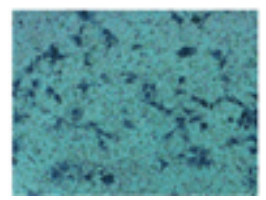

PDP NPS+LIFU
PDP NPs+LIFU
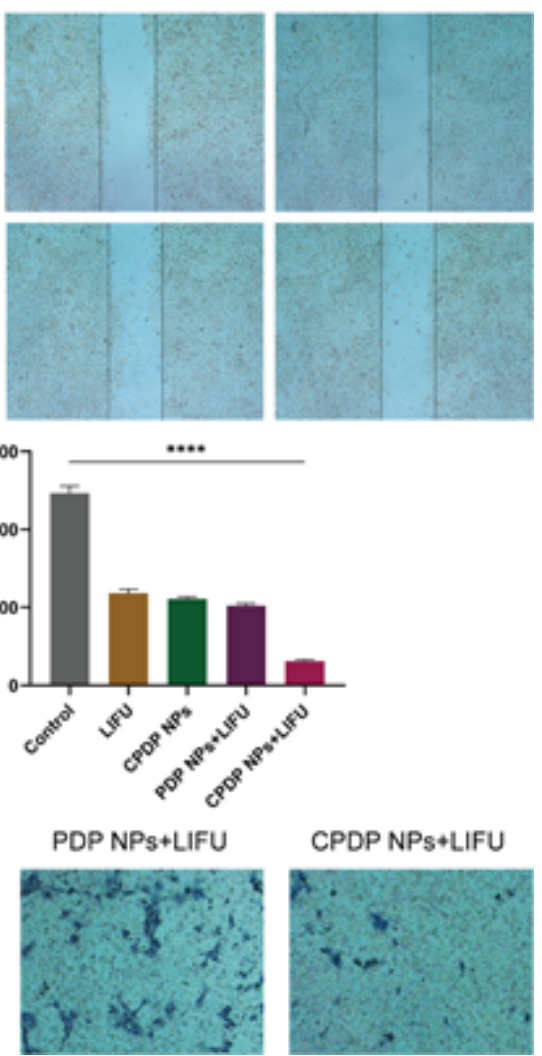

CPDP NPS+LIFU
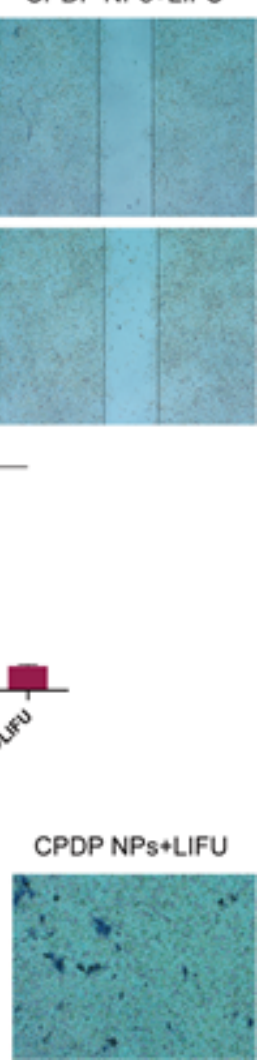

Figure 5 
(a) The wound healing and (b) The transwell assay after various treatments. (c) The corresponding

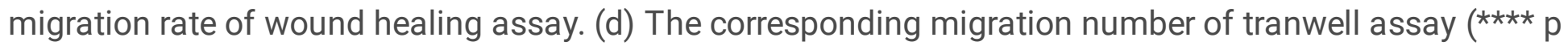
$<0.001, \mathrm{n}=3$ ).
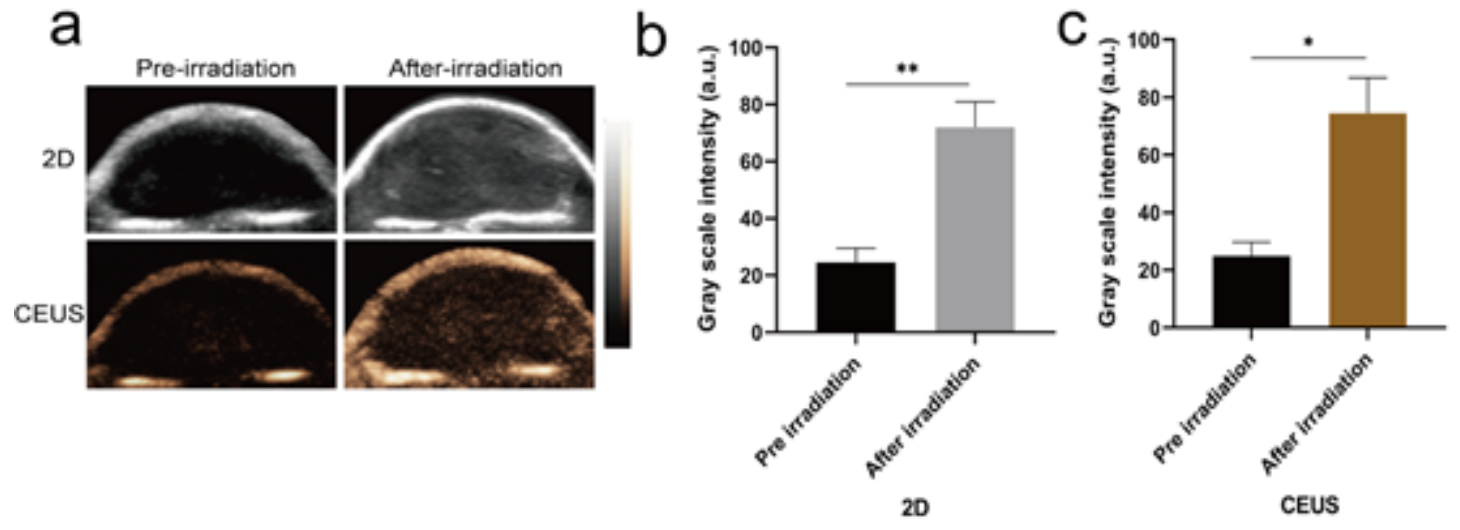

Figure 6

(a) 2D and CEUS images with and without LIFU irradiation. (b) and (c) The corresponding gray scale intensities analysis measured by ImageJ ( $\left.{ }^{* \star} p<0.01,{ }^{\star} p<0.05, n=3\right)$.
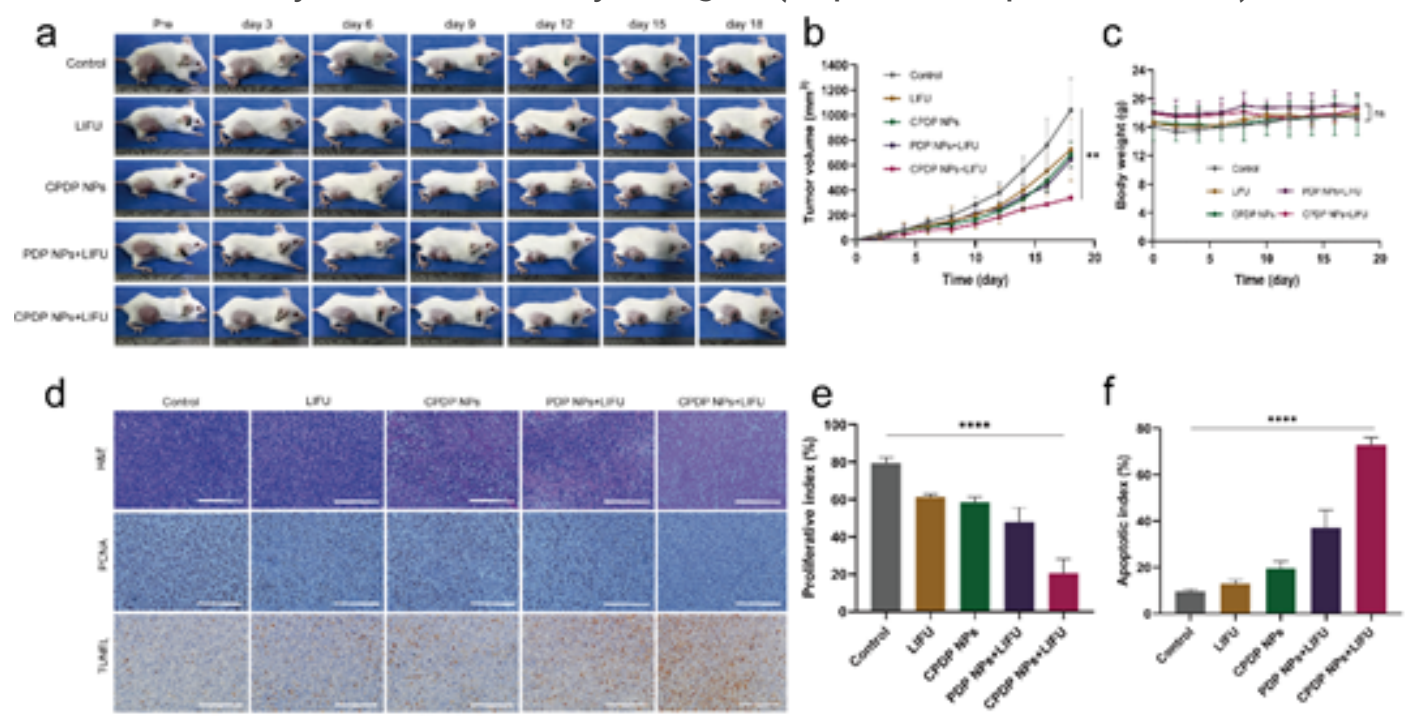

Figure 7

(a) Images of tumor-bearing mice under various treatments within the certain 18 days $(n=3)$. (b) Tumor volume analysis according to various treatments $(* \star p<0.01)$. (c) Weights of tumor-bearing mice under various treatments ( $\mathrm{ns}$ : no significance, $\mathrm{n}=3$ ). 

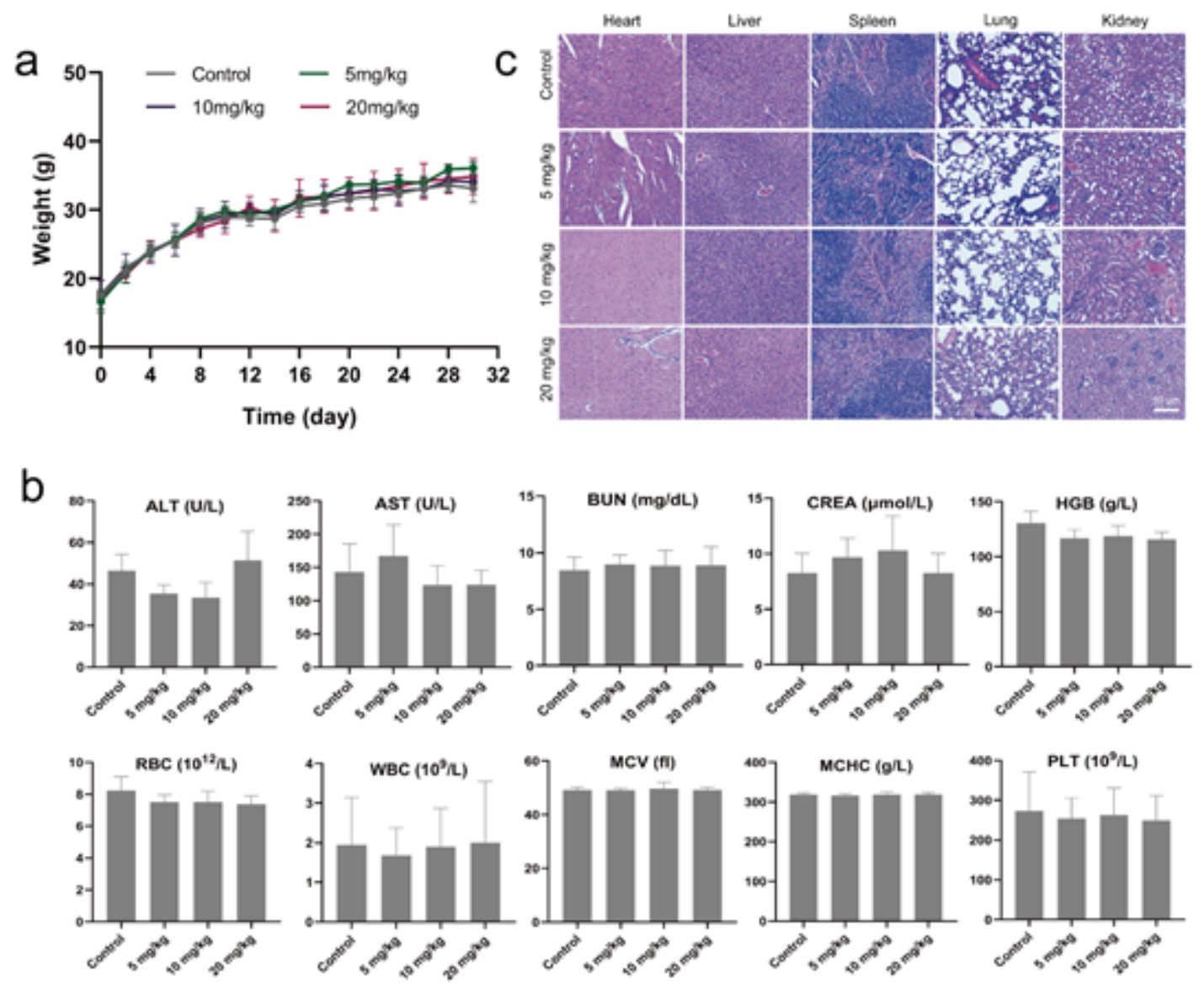

Figure 8

(a) The weights of healthy Kunming mice under various concentrations of CPDP NPs ( $n=3)$. (b) H\&E results of different organs (heart, liver, spleen, lung, and kidney) of mice under the same treatment (scale bar:50 $\mu \mathrm{m}$ ). (c) The blood biochemistry and blood routine examination under various concentrations of CPDP NPs within a certain period of 30 days $(n=3)$.

a

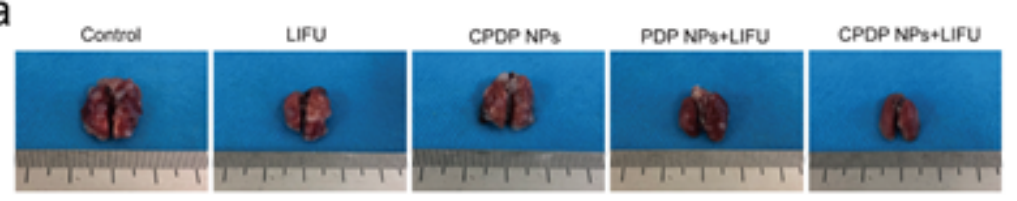

C
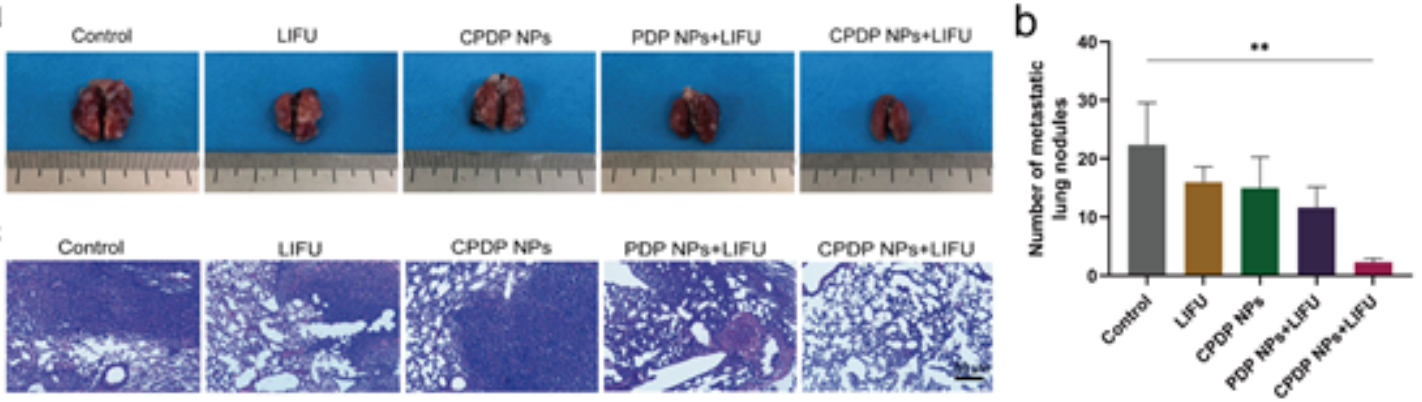

Figure 9

(a) Images of the general appearances of lung tissues. (b) The analysis of the metastatic lung nodules between various treatments (** $p<0.01, n=3$ ). (c) Corresponding images of lung metastatic H\&E staining results (scale bar:50 $\mu \mathrm{m}$ ). 